\title{
DERECHO Y ARQUEOLOGÍA EN EL SIGLO XIX: EL PRIMER ESTUDIO DE LAS TABLAS DE BRONCE DE MALACA Y SALPENSA*
}

\section{LAW AND ARCHAEOLOGY IN THE NINETEENTH CENTURY: THE FIRST STUDY OF THE BRONZE TABLETS OF MALACCA AND SALPENSA}

\author{
M. ${ }^{a}$ José Berlanga Palomo** \\ Universidad de Málaga
}

\begin{abstract}
RESUMEN. En este trabajo se aborda el primer estudio llevado a cabo en relación a uno de los descubrimientos más importantes en la Málaga decimonónica, dos tablas de bronce que contenían parte de las leyes municipales flavias de Malaca y Salpensa, respectivamente. El interés de su propietario, Jorge Loring, por conocer el contenido de sus textos va a motivar la incorporación de estudiosos ajenos al campo de las antigüedades, creándose una estrecha relación interdisciplinar entre materias, aparentemente tan distantes en aquel momento como el derecho y la arqueología. Este episodio será anterior al estudio, de gran éxito y alcance internacional, llevado a cabo por el jurista y epigrafista Manuel Rodríguez de Berlanga, cuya formación interdisciplinar le propiciará numerosos éxitos en el campo de la arqueología europea. Para ello, contamos con la información conservada en la Real Academia de la Historia, concretamente, con la correspondencia mantenida entre Ildefonso Marzo, correspondiente en Málaga y dicha institución, para recomponer la traducción y comentarios llevados a cabo por Miguel Téllez, abogado malagueńo, como primer acercamiento interdisciplinar a ambos documentos epigráficos.
\end{abstract}

PALABRAS CLAVE. Interdisciplinariedad, historiografía, arqueología, epigrafía, Malaca.

ABSTRACT. This paper addresses the first study carried out in relation to one of the most important discoveries in nineteenth-century Malaga, two bronze tables that contained part of the Flavial municipal laws of Malacca and Salpensa, respectively. The interest of its owner, Jorge Loring, to know the content of his texts will motivate the incorporation of scholars outside the field of antiquities, creating a close interdisciplinary relationship between subjects, apparently as distant at that time, such as law and archaeology. This episode will be prior to the study, of great success and international reach, carried out by the jurist and epigrapher Manuel Rodríguez de Berlanga, whose interdisciplinary training will lead him to many successes in the field of European archaeology. For this, we have the information kept in the Royal Academy of History, specifically, with the correspondence maintained between Ildefonso Marzo, corresponding in Malaga and said institution, to recompose the translation and comments carried out by Miguel Téllez, Malaga lawyer, as First interdisciplinary approach to both epigraphic documents.

KEYWORDS: Interdisciplinarity, historiography, archaeology, epigraphy, Malaca.

* Este trabajo se enmarca en el proyecto de investigación MINECO-Ministerio de Economía y Competitividad HAR2016-80271-P (IP M. Díaz-Andreu), subvencionado por la Agencia Estatal de Investigación (AEI) y el Fondo Europeo para el Desarrollo Regional (FEDER).

** Correspondencia a / Correspondence to: M. ${ }^{a}$ José Berlanga Palomo, Universidad de Málaga; Departamento de Ciencias Históricas mberlanga@uma.es - https://orcid.org/0000-0002-8376-246X.


y Salpensa", Veleia, 37, 33-44. (https://doi.org/10.1387/veleia.20980).

Recibido: 2 julio 2019; aceptado: 29 septiembre 2019.

ISSN 0213-2095 - elSSN 2444-3565 / (C) 2020 UPV/EHU 


\section{INTRODUCCIÓN}

Enterada la Real Academia de la Historia del descubrimiento fortuito, a finales de octubre de 1851, licitó a su correspondiente malagueño I. Marzo, que realizara copias y dibujos de las mismas para que la institución pudiera hacer su examen. También le pidió esta misma institución a Marzo que hiciese de intermediario con Jorge Loring, su propietario, para su destino, por compra, al Gabinete de Antigüedades. El interés de las dos tablas de bronce era el contener, respectivamente, parte de las leyes flavias municipales de las ciudades de Malaca y Salpensa. El propietario, sin embargo, no acepta tal propuesta, pero en julio de 1852 comunica a la Academia que está dispuesto a ofrecer las tablas para que sean estudiadas. Habrá que esperar finalmente hasta septiembre para su traslado por el compromiso que ha adquirido con D. Miguel Téllez y Sotomayor, abogado del Colegio de Málaga, que, según el mismo Jorge Loring, fue el primero que las estudió (Berlanga 2001).

Nos podemos preguntar por qué el propietario recurrió a un miembro de dicha institución, además de por amistad, como el ya mencionado. No cabe duda que este primer contacto de las tablas legislativas con el mundo del Derecho, que no será el único ni el más importante, pone de manifiesto la necesidad de recurrir a dicha disciplina para poder alcanzar un conocimiento efectivo de las mismas, cuyo valor quedaría mermado sin una correcta interpretación de los textos. Desde un primer momento, Jorge Loring entendió que un docto en leyes era el perfil más apropiado para esta tarea, por encima de otros personajes ilustrados que vivían en la Málaga de ese momento y a los que conocía por encuentros en espacios culturales como la biblioteca de los hermanos Oliver (Rodríguez de Berlanga 1903 (1995), 11-12). El objetivo principal de este trabajo será, por tanto, indagar el papel del Colegio de Abogados de Málaga, representado en Miguel Téllez y su colaborador José del Pino, con el primer estudio que se realizó de tales bronces, antes de la participación de un estudioso multidisciplinar como Manuel Rodríguez de Berlanga, cuyos estudios dieron a conocer las tablas legislativas a nivel internacional.

\section{El descubrimiento de los bronces, la Real Academia de la Historia y su vinculación A LOS LORING}

Manuel Rodríguez de Berlanga, cuyo estudio posterior de los bronces llegará a tener más peso que lo realizado anteriormente, nos explica detalladamente la aparición de estos bronces. Cuenta que se encontraron en las afueras de Málaga, por la parte norte en la zona conocida como los Tejares, llamados así por las fábricas de tejas y ladrillos que se abastecían de la arcilla allí existente a través de cortes en el terreno. En este lugar a finales de octubre de 1851 dos peones que cavaban para extraer la arcilla, encontraron dos tablas de bronce cuyos marcos se apoyaban sobre sendos espacios rodeados de grandes ladrillos romanos para cuidar su protección, con el anverso hacia el interior del hueco y el exterior cubierto por la arena que las cubría, quedando en una de ellas, la de mayor tamaño, restos de una cubrición con tela blanca (Rodríguez de Berlanga 1864, 333-337). También según este autor, ambos peones intentaron vender al peso el metal hallado, adquiriéndolas un industrial del metal que tenía situado su taller en la calle Compañía, un taller que en un plano conservado en el Archivo Histórico Municipal de 1847 aparece con el nombre de Casa del Belonero (Olmedo 2000, 24). Visitaba este taller un aficionado a las antigüedades, José Gálvez y González (Rodríguez de Berlanga 1873 (1995), 258), entusiasta admirador de los objetos de arte clásico, cuyo hijo, José Gálvez y Andújar, apoderado de Loring (Olmedo 2000, 23), le prestó igual 
servicio en la adquisición de los bronces de Osuna. Gálvez y González, enterado del hallazgo, rápidamente observó la particularidad y rareza de las planchas y, como su fortuna no le permitía comprarlas, avisó a Jorge Loring, quien rápidamente las compró y las trasladó a su casa en la Alameda (en 1861 todavía se podían ver en la casa de Jorge Loring en la Alameda no 26 (Vila 1861 (1998), 29 nota 2), siendo objeto de admiración por sus amigos y curiosos, y de difusión en la prensa local. Por ejemplo, el sábado 1 de noviembre de 1851 aparecía en el periódico El Correo de Andalucía sin firma de autor, un texto informando del hallazgo y características de las tablas, así como un primer acercamiento a su contenido (ver la reproducción de este texto en Rodríguez Oliva 2001, 10-12).

$\mathrm{Si}$, como acabamos de referir, las noticias sobre el hallazgo y estudio de las tablas loringianas se ha venido tradicionalmente, y con justificada razón, vinculando a la figura de Manuel Rodríguez de Berlanga (Rodríguez Oliva 2001; Rodríguez Oliva 2005), no obstante, las primeras noticias acerca del hallazgo de las dos tablas aparecieron ya pocos días después del mismo, cuando el joven erudito malagueño se encontraba en la Universidad Central de Madrid finalizando sus estudios de doctorado. Este hito significó también la vinculación y eterna amistad del erudito con una de las familias más poderosas de la burguesía malagueña, los Marqueses de Casa Loring (Campos 2006, 32-38; Ramos 2000, 53).

Es muy probable que la ampliación de los detalles del descubrimiento que va dando Rodríguez de Berlanga estén basada en la información dada por otro erudito malagueño, Ildefonso Marzo, a la Real Academia de la Historia, sobre la que vamos a basar nuestros comentarios.

\section{INTERDISCIPLINARIEDAD EN EL ESTUDIO DE LOS BRONCES LORINGIANOS}

Ildefonso Marzo tendría un papel protagonista en los primeros momentos del descubrimiento. Nació en Alhaurín el Grande en 1794, pero inició sus estudios en Málaga. Fue Capitán de Guardias de Corps, y tras retirarse, fue nombrado escribano de su pueblo, cargo que desempeñó hasta su muerte, acaecida en 1856. Desde joven, sus poemas, sus escritos históricos y arqueológicos y sus disertaciones filosóficas eran conocidos en las tertulias de los intelectuales malagueños. Prueba de su participación en la vida cultural e intelectual malagueña fue su participación en el acto de inauguración del Liceo de Málaga, siendo uno de los miembros fundadores. Su obra principal, a la que dedicó los últimos años de su vida, fue su Historia de Málaga y su provincia, que quedó inconclusa, publicándose dos tomos en 1850 y 1851, de los tres que tenía pensado escribir. En la portada del primero se presenta como «miembro de la Junta de Arqueología y Numismática de la Biblioteca Nacional en la provincia de Málaga» (Pérez 2015; Rodríguez 1986, 193-196).

Desde 1845 Ildefonso Marzo era miembro correspondiente de la Real Academia de la Historia y, como tal, fue el que informó a esta institución del importante hallazgo de las tablas de bronce en noviembre de 1851. Pocos días después del descubrimiento, Marzo informaba a la Institución del hallazgo, remitía un ejemplar de la noticia aparecida en el Correo de Andalucía y recibía de esta la petición de hacer copias y dibujos para que los académicos pudiesen realizar su examen. El malagueño ya había solicitado al dueño de las tablas, Jorge Loring, la posibilidad de estudiarlas, a lo que aquel no puso ningún impedimento, dedicándose a partir de ese momento a hacer una copia y estudio de los textos contenidos en las mismas (Archivo RAH, CAMA/9/7962/6(2)). Los datos aquí conservados nos podrían hacer pensar que la información dada en el Correo de Andalucía el 1 de noviembre de 1851 acerca del descubrimiento y contenido de las tablas pudo haber sido transmitida por el propio Marzo. Sin embargo, sus comentarios hacia el mismo confirman lo contrario: 
«Pero al principiar este trabajo pude juzgar por mí mismo que los pocos aficionados al estudio de las antigüedades que cuenta la ciudad de Málaga solo habían llegado a trasladar en letra cursiva y común, columna y media del contenido de la tabla mayor, no obstante de haberse hecho cargo, a primera vista, de todo el sentido de ambos bronces. Y como el público ilustrado, que sabía el descubrimiento, se hallase con grandes deseos de saber sus referencias, creyeron muy oportuno estos investigadores escribir un artículo acerca de su importancia en el diario malagueńo titulado El Correo de Andalucía, dando algunos pormenores relativos a su descubrimiento con otras explicaciones a la verdad precipitadas y sin aquella justa necesidad de un examen concienzudo y de un análisis detenido.» (Archivo RAH, CAMA/9/7962/6(4))

Estas primeras noticias de Ildefonso Marzo a la Real Academia de la Historia ponen de manifiesto la dificultad que suponía, incluso para los más doctos en las antigüedades de la ciudad, hacer frente a un estudio serio y exhaustivo de los textos; era necesario la aportación de otra disciplina, la jurídica. De hecho, si observamos las noticias dadas en esa primera información a la prensa, se basan en datos de carácter físico y está ausente cualquier intento de traducción seria, conformándose el público con la identificación, errónea en parte, «de que estas tablas contienen las leyes locales del Municipio de Málaga en los primeros años del imperio romano» (Archivo RAH, CAMA/9/7962/6(4)). En este mismo oficio, enviado por Marzo a Pedro Sabau y Larroya, Secretario Perpetuo de la Real Academia de la Historia, el 14 de enero de 1852, además de remitir un primer estudio de las tablas de bronce descubiertas en Málaga, informa con más detalle de las circunstancias del hallazgo con una disertación sobre su contenido, cronología y autenticidad, pero debido a su falta de formación en este campo, muy lejos de poder considerarse un estudio serio (Archivo RAH, CAMA/9/7962/6(4)). Al mismo tiempo, informa que no solo se ha dedicado al estudio de los textos, sino que también se ha interesado en

«examinar con detención la superficie de las tablas y ese barniz de vetustez que no puede equivocarse y que solamente se forma bajo el influjo de tiempo. En los claros que dejan las letras, domina en el bronce de Málaga el óxido rojo de los ańos, el que raspado cuidadosamente resistía desaparecer, sobre todo no estando herido por el óxido verde que se conocía por más reciente y auxiliándome en esta operación y examen el acreditado químico y naturalista Don Pablo Prolongo, oyendo sus concluyentes razones sobre el estado físico y aparente de la superficie de los bronces, no me quedó la menor duda de la autenticidad del monumento.» (Archivo RAH, CAMA/9/7962/6(4))

Resulta interesante que Don Pablo Prolongo, prestigioso farmacéutico y boticario de la ciudad, corresponsal nacional de la Academia Real de Ciencias de Madrid y miembro fundador de la Sociedad Malagueña de Ciencias (Castellanos), también aportase los conocimientos vinculados con su disciplina, lo que nos confirma, una vez más, que ya en esos momentos resultaba bastante evidente que para un estudio completo de las piezas era necesario una labor interdisciplinar. Concluye con algunos datos acerca del lugar exacto del hallazgo (figura 1), ubicándolo concretamente en el sitio llamado de los Ejidos, cerca de la hacienda de Don Domingo Orueta. También, según su opinión, las tablas que faltaban de Malaca y Salpensa habían sido encontradas previamente y fundidas, ya que el dueño de la finca donde aparecieron, algún tiempo antes, tropezó el arado con unos ladrillos romanos, que por su descripción podrían ser de la misma solería que había bajo las tablas. 


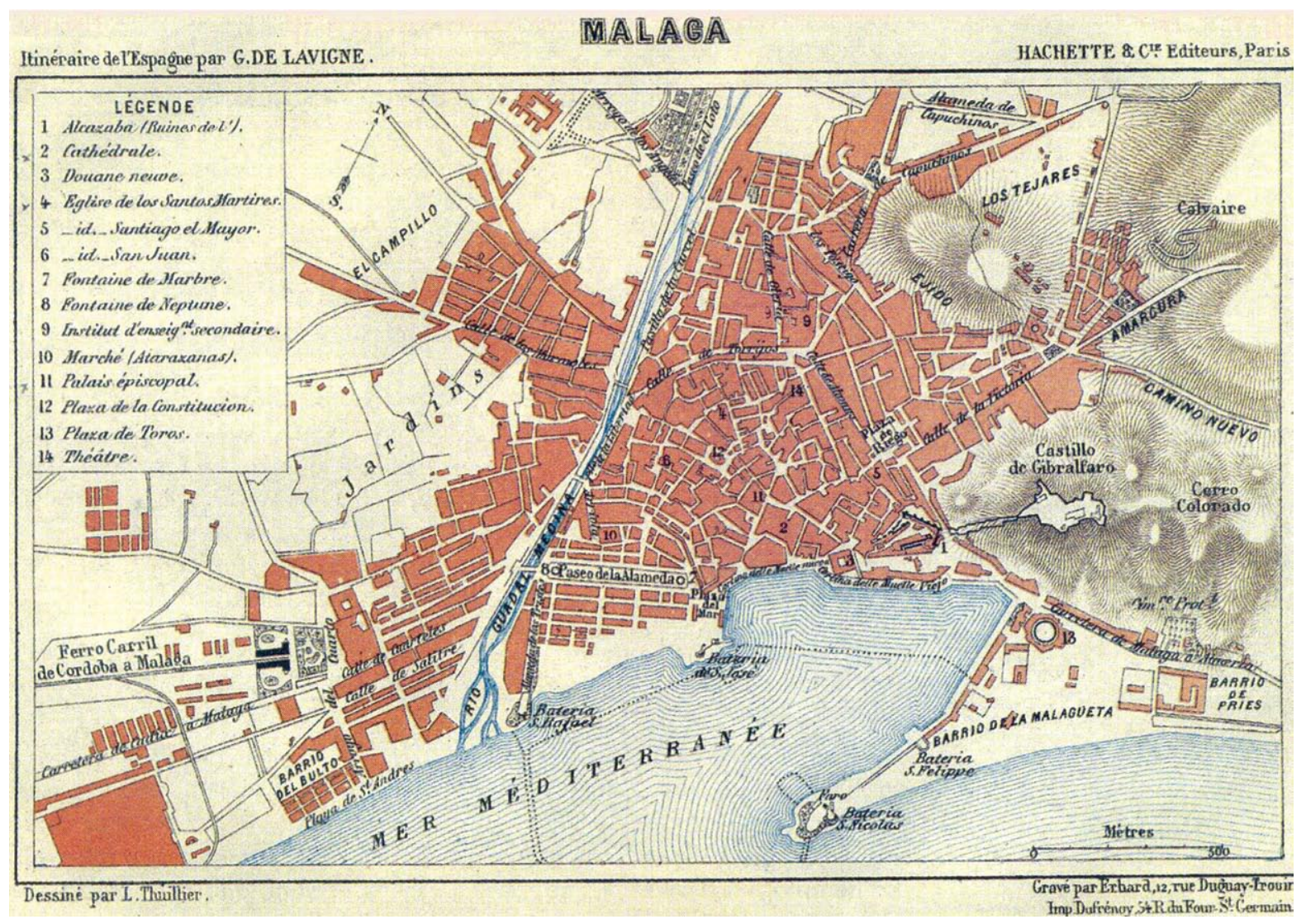

Figura 1. Mapa de Málaga (Thuiller 1880).

Parece evidente y, al mismo tiempo extraño, que Domingo Orueta y Aguirre no había tenido conocimiento del hallazgo de otros fragmentos pertenecientes a otras tablas de bronce que acompañarían a las dos descubiertas en 1851, como tampoco de este hallazgo. Conocida es su gran afición al coleccionismo de antigüedades, sobre todo prehistóricas (Guillén 1874 (1983), 11 nota 1). Prueba de su interés por los hallazgos arqueológicos de la provincia es, por ejemplo, la visita que realiza con Berlanga en la exploración de las termas descubiertas en Torremolinos (Rodríguez de Berlanga 1861,2). Orueta y Aguirre es principal partícipe en la creación de la colección arqueológica de la Sociedad Malagueña de Ciencias, de la que fue principal fundador y repetidas veces presidente. También mostró una gran afición a la geología y fue autor, entre otros trabajos, de Los barros de los Tejares (Orueta 1874), en el que, al analizar los estratos que componen esta formación geológica, aparece una breve referencia al descubrimiento:

«En el piso superior existe en primer lugar un fango moderno, de un metro poco más o menos de profundidad, donde fueron encontradas las célebres Tablas de bronce, referentes a los municipios de Malaca y Salpensa durante la dominación romana, que se ven hoy en la mencionada hacienda de los Sres. Marqueses de Casa-Loring y que tan hábilmente han sido interpretadas por mi buen amigo y distinguido arqueólogo, el Sr. D. Manuel Rodríguez de Berlanga.» (Orueta 1874, 4-5) 
No cabe duda de que, de haber existido en estos momentos, la Sociedad Malagueña de Ciencias habría tenido un papel fundamental en el examen y estudio de las Tablas Loringianas, pues se puede considerar la institución más interdisciplinar de la Málaga decimonónica. Ciertamente, a mediados del siglo XIX se consolida en Málaga una burguesía socio-económica que se acercará a los intelectuales prestando un decidido mecenazgo. En este contexto, el 24 de julio de 1872, una veintena de malagueńos, convocados por Domingo de Orueta y Aguirre, celebran una reunión preparatoria para crear la que poco más tarde (6 de octubre de 1872) se constituiría formalmente como Sociedad Malagueña de Ciencias Físicas y Naturales. Entre los socios fundadores se encontraban médicos, científicos, botánicos, matemáticos, y también un arabista, J. Guillén Robles. Un año antes se había constituido la Real Sociedad Española de Historia Natural, que contaba entre sus socios fundadores con Amalia Heredia, Marquesa de Casa Loring (Perejón 2008, 5).

La actividad de la academia en el ámbito arqueológico podemos rastrearla a través de las conferencias, discursos e informes, recogidos en su boletín y las actas, que nos permiten comprender la interacción entre las otras ciencias y la arqueología. El interés por esta está presente desde su origen, pues como queda recogido en el acta de la sesión del 6 de octubre de 1872: "Se aceptó en principio la idea expuesta por el Sr. Guillén (Robles) de que la Sociedad de Ciencias Naturales y Físicas extendiese su esfera a la Arqueología» (Sociedad Malagueńa de Ciencias Físicas y Naturales 1874, 4). Poco después, en el Acta de la Sesión del 13 de octubre de 1872: «se recordó por uno de los socios presentes que el Sr. Guillén y Robles había propuesto ampliar el Museo a los objetos arqueológicos» (Sociedad Malagueña de Ciencias Físicas y Naturales 1874, 4). No cabe duda que la labor e insistencia de Guillén Robles y otros miembros, como el propio Presidente, Domingo Orueta y Aguirre, hizo que las humanidades tuviesen su hueco en la Sociedad. De hecho, hoy día forma parte de una de sus secciones (Álvarez 2015; Carrillo, Ramos y Castellanos 1984; Prolongo 1895).

Volviendo al momento de su descubrimiento, y gracias de nuevo a la información aportada por Ildefonso Marzo (Archivo RAH, CAMA/9/7962/6(4)), sabemos que la Comisión de Monumentos, el ayuntamiento y el gobernador de la provincia, conscientes del importante hallazgo, reclamaron su adquisición, a lo que Loring contestó que no había inconveniente en cederlas a la Comisión de Monumentos, cuando esta tuviese un local o museo donde pudieran ser trasladadas. Jorge Loring conocía perfectamente el negativo funcionamiento de la Comisión de Monumentos, a la que pertenecía desde su creación en 1844 el padre de la Marquesa de la Casa-Loring, Manuel Agustín Heredia (Archivo Real Academia de Bellas Artes de San Fernando, signt. 39/3-2(5)), y los fracasos de esta por hacerse con un local para sede del inexistente Museo Arqueológico de Málaga (Berlanga 2006, 73-77). En otro oficio enviado por Idelfonso Marzo a Antonio Delgado y Hernández, Secretario de la Sección o Comisión de Antigüedades de la Real Academia de la Historia, varios meses después, el 28 de septiembre de 1852, se especifica que los autores de la traducción de las tablas enviada a la institución han sido Miguel Téllez y Sotomayor y José del Pino, ambos miembros del Colegio de Abogados de Málaga (Archivo RAH, CAMA/9/7962/6(9)). De las visitas que recibió el matrimonio Loring para estudiar las tablas, también nos dejó su particular y sincera opinión M. Rodríguez de Berlanga:

«Cuando los que las descubrieron se cercioraron que eran de cobre procuraron venderlas al peso, habiéndolas adquirido un constructor de objetos de dicho metal, que estaba establecido en la calle de la Compañía. Frecuentaba el obrador de aquel industrial un sujeto [José Gálvez y González] algo dado a coleccionar cosas antiguas y no bien tuvo noticias de la adquisición de aquellas dos planchas y las hubo visto adivinó su rareza, sin comprenderla y se interesó por salvarlas del peligro 
inmediato de ser fundidas, que las amenazaba. Su modesta fortuna no le permitía realizar por sí tan elevado propósito; pero no dudó un momento que el Marqués de Casa-Loring habría de ser el único que en Málaga respondiera satisfactoriamente a su excitación, en lo que no se engañaba por cierto. Tener noticia de lo acaecido, ir a examinar el hallazgo, convenir con el que lo poseía el precio que interesaba por su venta, dejándolo que realizase no exigua ganancia, abonarle lo estipulado y trasladar a su casa una y otra tabla, donde las esperaba con impaciencia la Marquesa, fue obra de brevísimo tiempo.»

La casa de los Loring se encontraba desde 1851 en la Alameda no 26 (Ramos 2000, 40). Sobre el tiempo breve del que habla el documento hemos de decir que, en efecto, el 1 de noviembre, fecha en la que aparece la noticia del descubrimiento en El Correo de Andalucía, se dice en esta que ya han sido adquiridas las dos tablas por su nuevo propietario (Rodríguez Oliva, 2001, 16). El documento sigue así:

«Los periódicos de la localidad dieron detallada cuenta de tan inesperado descubrimiento y de qué manera providencial habíase librado de la destrucción que les aguardaba, con lo que bien pronto, los amigos más íntimos primero y las personas más caracterizadas después acudieron a casa de los Marqueses a satisfacer su curiosidad, de que no pudo sacarles la simple inspección de aquellos monumentos enteramente mudos para ellos.» (Rodríguez de Berlanga 1903 (1995), 10)

Entre esas personas «caracterizadas» se encontraban el propio Ildefonso Marzo, al que puede referirse el comentario crítico de Berlanga, quien dejó constancia en numerosos de sus escritos de su visión negativa hacia la producción histórica de Marzo. Del artículo de Marzo sobre Cartima critica la copia inexacta de las inscripciones y la mala traducción (Rodríguez de Berlanga 1861, 180181) y su Historia de Málaga Berlanga la califica como «Obra escrita con depravado gusto literario sin crítica y con tanta falta de conocimientos que la hacen de peor condición que las Conversaciones Históricas Malagueñas» (Rodríguez de Berlanga 1861, 182).

Enterada la Real Academia de la Historia del descubrimiento, también solicitó a Ildefonso Marzo, junto a la realización de copias y dibujos de las mismas para que la institución pudiera hacer su examen, que hiciese de intermediario con Jorge Loring para su destino, por compra, al Gabinete de Antigüedades (Archivo Secretaría RAH, expediente personal de Ildefonso Marzo). Como hemos apuntado anteriormente, su propietario no acepta tal propuesta y ya hemos comentado que en julio de 1852 comunica a la Academia que está dispuesto a ofrecer las tablas para que sean estudiadas, pero, también como ya hemos aludido al principio de este trabajo, habrá que esperar hasta septiembre para su traslado por el compromiso que ha adquirido con D. Miguel Téllez y Sotomayor, abogado del Colegio de Málaga. Este, según el mismo Jorge Loring, fue el primero que "descifró sus caracteres» y las estudió (figura 2) (Archivo RAH, CAMA/9/7962/6(9) y CAMA/9/7962/6(5)). Don Miguel Téllez de Sotomayor Navarro era hijo de una ilustre y antigua familia malagueña. Fue abogado y propietario de numerosas casas y fincas y en 1852 se casó con dońa Isabel Conti y Conti, rica propietaria nacida en Granada. Ambos vivieron en La Alameda 29. Su hermano, Joaquín Téllez de Sotomayor, reconocido Ingeniero de Canales, Caminos y Puertos ingresó el 21 de noviembre de 1850 en la Real Academia de Bellas Artes de San Telmo de Málaga, junto a Jorge Loring, que recibirá el cargo de Tesorero (realacademiadesantelmo.org). En 1848, Miguel Téllez se incorporó al Ilustre Colegio de Abogados de Málaga, siendo decano los años de 1864 y 1872-73. Falleció en Madrid en 1878 (Colegio de Abogados de Málaga 1887, 2-17). 
La traducción debió de realizarse en el intervalo de ambos escritos, es decir, entre el 30 de enero y el 28 de septiembre de 1852, justo antes del estudio de Rodríguez de Berlanga, pues en el primero Ildefonso Marzo nos informa que

«hasta ahora no han sido traducidas, de ello se ocupan algunos curiosos con más o menos confianza y algunos presuntuosos o semisabios con enfática petulancia. Yo confieso que es empresa de mucho estudio y detenimiento y muy superior a mis fuerzas por más que ello me estimulen mis particulares deseos y mi afición a estos estudios.» (Archivo RAH, CAMA/9/7962/6(4))

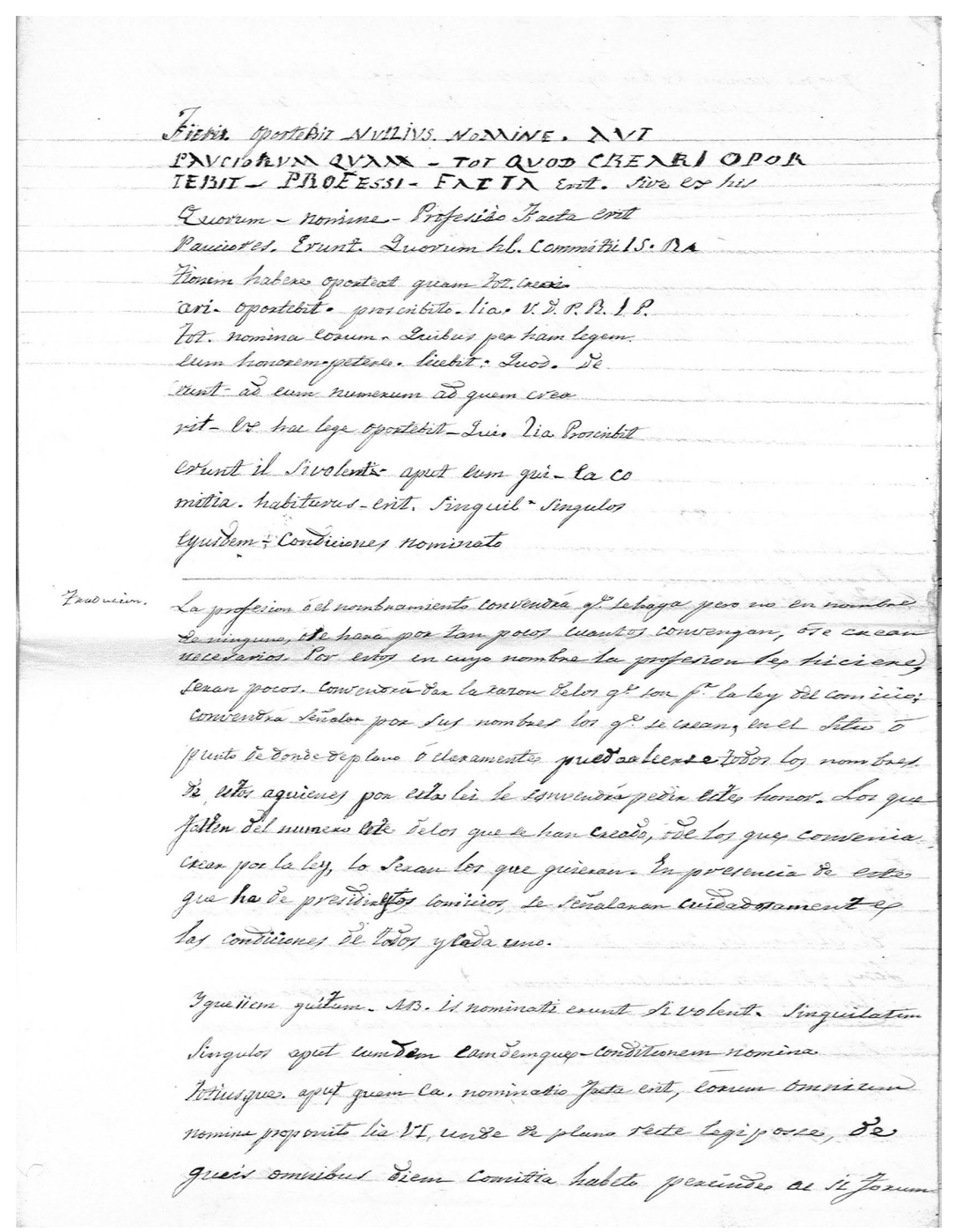

Figura 2. Escrito de Ildefonso Marzo en el que se cita a los abogados Miguel Téllez y José del Pino como autores de la primera traducción de las Tablas loringianas (Archivo RAH, CAMA/9/7962/06(5)).

Si analizamos los 28 folios que componen esta primera traducción, el método elegido por su traductor ha consistido en una transcripción del texto latino por epígrafes, acompañado de un comen- 
tario-traducción cuyo principal objetivo es que sea comprensible a un público no experto. Podemos decir, que la labor de Miguel Téllez, tiene, además, una clara intención divulgativa, tratándose más de una traducción para ese público culto curioso y deseoso de conocer el contenido de los bronces que un estudio exhaustivo y erudito, como veremos más tarde en el trabajo de Rodríguez de Berlanga (figura 3). Finalmente, habrá que esperar a agosto de 1855 para que Ildefonso Marzo informe a la Academia de que Jorge Loring no tiene inconveniente en su traslado a Madrid para que sean estudiados los dos ejemplares, si bien no será hasta 1857 cuando, finalmente, Jorge Loring, en uno de sus viajes a Madrid, envíe solo una de las tablas, la de Salpensa, a la Real Academia de la Historia, donde permaneció el mes de junio y primeros días de julio, momento en que fue reclamada por su propietario (Archivo RAH, CAMA/9/7962/10). Sin embargo, aunque la Academia solicitó en numerosas ocasiones, ya desde 1852, un estudio por parte de la Comisión de Antigüedades, formada en esos momentos por Marcial Antonio López, como presidente (sustituido en 1857 por Pascual Gayangos) y Antonio Delgado, José Amador de los Ríos y Pedro Sabau (este último hasta 1856), como vocales (Maier 1998, 41), este nunca se hizo efectivo, lo que pone de manifiesto una vez más la necesidad de una interdisciplinariedad que no podía ofrecer la institución. Sí consta un informe elaborado por A. Delgado y P. Gómez de la Serna, quien se ocupaba de emitir pronunciamientos legales sobre los asuntos controvertidos que llegaban a la Academia, sobre el facsímil de la Lex Salpensana que enviara M. Rodríguez de Berlanga (CAMA/9/7962/10 (14); (Ruiz 2013, 278-279).
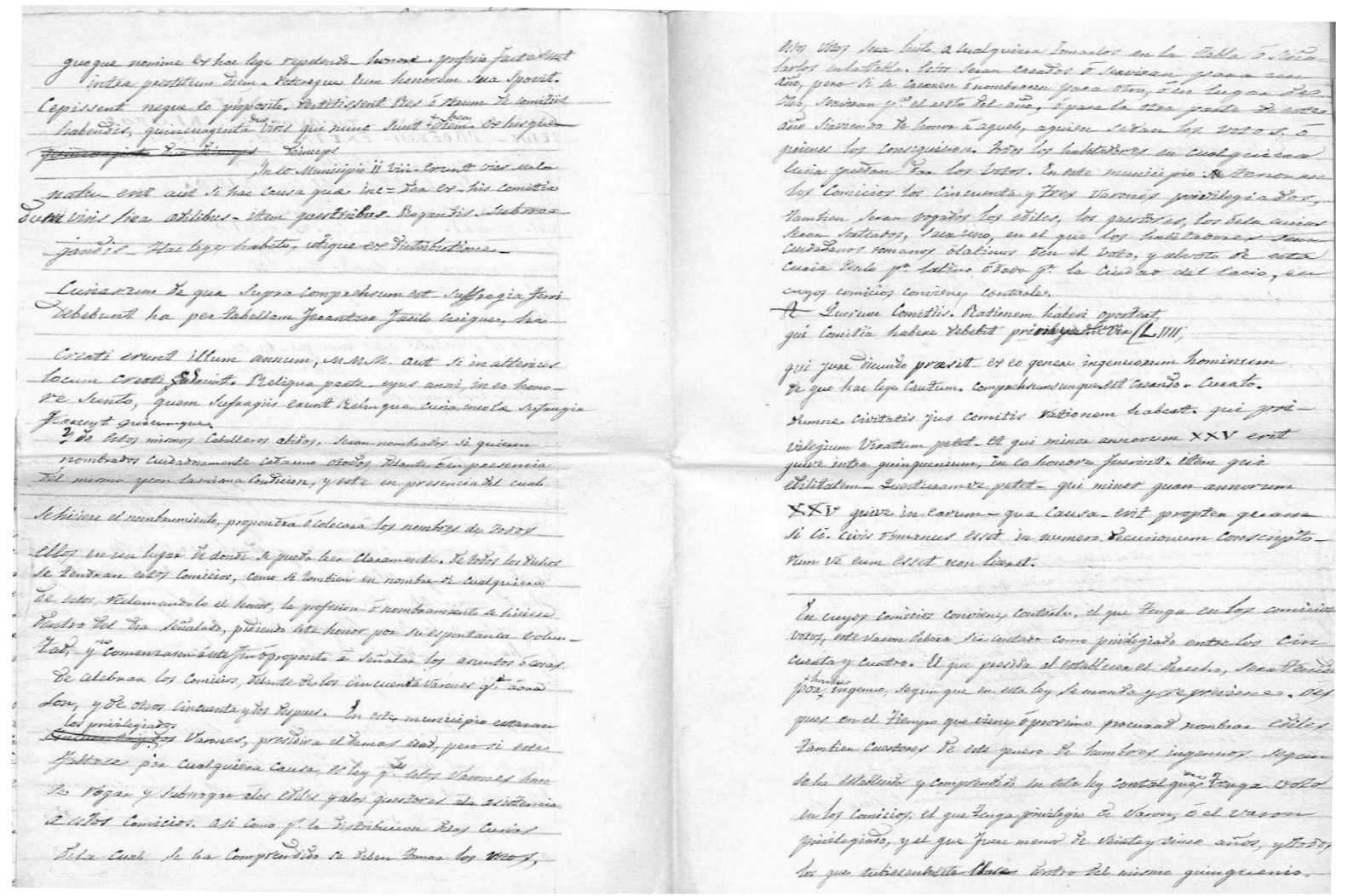

Figura 3. Páginas 6-7 de la traducción realizada por los abogados Miguel Téllez y José del Pino (Archivo RAH, CAMA/9/7962/06(9)). 


\section{Destino Final de los bronces loringianos}

Es en ese mismo año de 1855, cuando se reorganiza la Comisión de Monumentos Históricos y Artísticos, de la que serán miembros el propio Ildefonso Marzo, junto a Jorge Früller (vicepresidente), Manuel Rodríguez de Berlanga (secretario), Cirilo Salinas (arquitecto provincial), Jorge Loring, y Diego Montant (Archivo Real Academia de Bellas Artes de San Fernando, signt. 49/32(5)). A finales de este ańo Jorge Loring lleva a cabo la compra de los terrenos de la Hacienda de la Concepción, que pasaría a convertirse en lugar de recreo de la familia, jardín botánico y museo arqueológico. La refinada cultura del matrimonio Loring, de la que Berlanga (Rodríguez de Berlanga 1903 (1995), 9) nos recuerda que en su viaje de bodas recorrieron Francia, Italia, Suiza y Alemania — siendo su primer objetivo la visita a las bibliotecas, museos arqueológicos, de pintura y los monumentos arquitectónicos, y ahora, la propiedad de los bronces-, crearon las circunstancias propicias para la creación del museo. Es poco después de la adquisición de la Hacienda, en mayo de 1858, cuando Jorge Loring solicita a la Real Academia de la Historia permiso para adquirir la propiedad de todas las antigüedades que encuentre en la provincia, petición, que, ante la inexistencia de una legislación que regule estas actividades, le fue denegada (Archivo RAH, CAMA/9/7962/12). A pesar de esta negativa, el matrimonio Loring y, especialmente, Amalia van a emprender una labor de recolección de objetos arqueológicos dispersos por la ciudad y provincia, y Rodríguez de Berlanga, al lado del matrimonio durante la creación de esta colección arqueológica, será el encargado de realizar un primer catálogo en 1868. El espacio elegido para albergar la colección fue un templete de orden dórico mandado hacer expresamente, según nos dice Hübner, al arquitecto alemán Wilhelm Strack (CIL II, Suppl, 876). Como pavimento del templete se escogió un mosaico decorado con los doce trabajos de Hércules descubierto en Cártama en 1858 (Rodríguez 2001, 32). No obstante, las tablas de Malaca y Salpensa permanecieron, según la Guía de la Provincia de Málaga de Benito Vilá, al menos hasta 1861, en la residencia de la Alameda (Vila 1861 (1998), 29 nota 2 ).

No conocemos el momento en el que son trasladadas al Museo de la Finca de la Concepción, pero allí permanecieron hasta 1897, exceptuando su salida para dos exposiciones artísticas celebradas en Málaga, la primera organizada por el Liceo de Málaga en junio de 1874 y otra en 1881. En ambas las antigüedades suponían una pequeña representación en el total de la exposición, pero son una interesante fuente de información para conocer a algunos de los coleccionistas de antigüedades de la capital (Catálogo 1874; Muñoz 1881).

Conocida la experiencia de la colección Villacevallos, comprada por los marqueses en un lamentable estado de abandono (Rodríguez de Berlanga 1903 (1995), 149-156) y otras que corrieron igual suerte, los marqueses se preocuparon al final de sus vidas por el futuro de su colección, especialmente por los bronces jurídicos. La solución fue la venta al Museo Arqueológico Nacional. Por Real Decreto de 23 de julio de 1897, firmado por la Reina Regente María Cristina, el Estado adquiría para el Museo Arqueológico Nacional, del que era director Juan de Dios de la Rada y Delgado, las seis tablas de bronce al precio de 100.000 pesetas (Mélida 1897).

\section{Conclusiones}

Resulta evidente que la investigación en torno a las tablas de bronce que contenían fragmentos de las leyes municipales flavias de Malaca y Salpensa, inmediatamente después de su hallazgo, puso de relieve que este tipo de material arqueológico no podía ser abordado únicamente por espe- 
cialistas en materia arqueológica. Las fuentes directas que se han conservado, especialmente la correspondencia con la Real Academia de la Historia, nos transmiten que hubo personalidades del ámbito malagueño, de muy diversa formación, que desearon aportar su conocimiento para dar luz a los textos y al soporte en el que se elaboraron. Entre ellos, jugaron un papel importante aquellos con formación letrada, siendo los más aptos para tan difícil empeño, pues darían a su propietario, por otra parte, el verdadero valor de su reciente adquisición. La elección de Jorge Loring de ceder el estudio de las tablas al abogado Miguel Téllez pudo deberse a diferentes motivos, entre ellos, la amistad, y, sobre todo, la ausencia en la ciudad en esos momentos de otra personalidad más apta para el empeño. Es cierto que su traducción no dio los resultados más satisfactorios, pues la única versión que conocemos de su trabajo es la enviada a la Real Academia de la Historia, donde quedó archivada sin mayor difusión. A pesar de ello, hay que valorar la implicación de este abogado, totalmente ajeno a la Arqueología, como primera aportación interdisciplinar en el estudio de estos monumentos históricos.

El éxito definitivo en el estudio de las tablas de bronce vendría con la aportación de un personaje que reunía todos los requisitos necesarios, estudios jurídicos y humanísticos, Manuel Rodríguez de Berlanga. Como avanzábamos, durante su estancia en la Universidad Central de Madrid, fue informado del descubrimiento y animado por el rector de la Universidad, el marqués de Morante, de que iniciara el estudio del texto contenido en ambas tablas (Rodríguez de Berlanga 1903 (1995), 14). De vuelta en Málaga, conoció a Jorge Loring en una de las reuniones que se celebraban en la biblioteca de los hermanos Oliver y el joven propietario de las Leyes puso a su disposición todas las facilidades para que las estudiase. El resultado de este trabajo, realizado entre noviembre de 1852 y enero de 1853, fue la constatación de que los textos grabados en uno y otro bronce correspondían a fragmentos de sendas constituciones de Municipios Flavios de Malaca y Salpensa, y la publicación de la transcripción de ambos textos en una revista local titulada Revista Semanal Pintoresca del Avisador Malagueño, de la que envió una tirada aparte a Italia y Alemania, lo que supuso el conocimiento y estudio a nivel europeo de ambos monumentos de la antigüedad y, al mismo tiempo, el reconocimiento de la labor de Rodríguez de Berlanga entre los estudiosos de la antigüedad (Carl Gottlob Zumpt, Theodor Mommsen, Johann Heinrich Wilhelm Henzen, Pietro Capei y otros), mientras que en España su labor era reconocida con la concesión del título de Caballero de la Real y distinguida Orden de Isabel la Católica el 9 de abril de 1853 (Rodríguez de Berlanga 1903 (1995), 15-18; Rodríguez 1991, 100; Rodríguez 2005).

\section{BiBLIOGRAFÍA}

Álvarez, M., 2015, Anotaciones históricas sobre la Sociedad Malagueña de Ciencias (1872-2002). Málaga: Academia Malagueña de Ciencias.

Berlanga, M. J., 2001, «El descubrimiento de las tablas de bronce con las leyes municipales de Malaca y Salpensa en Octubre de 1851», Jábega 88, 54-62.

Berlanga, M. J., 2006, Arqueología y erudición en Málaga durante el siglo XIX. Málaga: Universidad de Málaga.

Carrillo, J. L., M. D. Ramos \& J. Castellanos, 1984, La Sociedad Malagueña de Ciencias. Catálogo de sus manuscritos, Málaga: Servicio de Publicaciones de la Universidad de Málaga.

Castellanos, J., «Pablo Prolongo García». Real Academia de la Historia, Diccionario Biográfico electrónico (https://dbe.rah.es/).

Catálogo... 1874, Catálogo de la Exposición Retrospectiva celebrada por El Liceo de Málaga en el mes de junio de 1874, Málaga: Imprenta y Librería de Rubio y Cano, sucesores de Martínez de Aguilar. 
Colegio de Abogados de Málaga, 1887, Lista de los abogados del Ilustre Colegio de la Ciudad de Málaga. Año económico de 1887-1888, Málaga: Imprenta de M. Rando Navas.

GuIllén, F., 1874 (1983), Historia de Málaga y su provincia [Edición facsímil], Málaga: Arguval.

Maier, J., 1998, Comisión de Antigüedades Comunidad de Madrid. Catálogo e indices [Publicaciones del Gabinete de Antigüedades, Serie 4: Documentos; 4, 1], Madrid: Real Academia de la Historia.

Mélida, J. R., 1897, «Museo Arqueológico Nacional. Bronces de Osuna de Málaga, de Salpensa y de Bonanza", Revista de Archivos, Bibliotecas y Museos (3. a época) 1, 522-524.

Muñoz, N., 1881, «La Exposición Artística en Málaga», La Ilustración Española y Americana X, 260, 262.

Olmedo, M., 2000, «Introducción», en: M. Rodríguez de Berlanga (ed.), Monumentos Históricos del Municipio Flavio Malacitano [Edición facsímil], Málaga: Servicio de Publicaciones Centro de Ediciones de la Diputación Provincial de Málaga, 9-129.

Orueta, D., 1874, Los barros de los Tejares, Málaga: Imp. Económica.

Perejón, A. (ed.), 2008, Boletín de la Real Sociedad Española de Historia Natural. Actas, Tomo 105. Madrid: Facultades de Biología y Geología, Ciudad Universitaria.

Pérez, S. D., 2015, La obra de Ildefonso Marzo y Sánchez. La Historiografía malagueña de la primera mitad del siglo XIX, Málaga: Tesis Doctoral. Universidad de Málaga.

Prolongo, A., 1895, Datos biográficos de D. Domingo Orueta y Aguirre leídos ante la Sociedad Malagueña de Ciencias Físicas y Naturales por el socio D. Agustín Prolongo y Montiel el 21 de noviembre de 1895, Málaga: Impr. y Lit. de Ramón Párraga.

Ramos, E., 2000, Amalia Heredia Livermore, Marquesa de Casa-Loring. Málaga: Servicio de Publicaciones e Intercambio Científico de la Universidad de Málaga.

Rodríguez, I., 1986, «Ildefonso Marzo», en: M. Alcobendas Francis (ed.) Málaga. Personajes en su historia, Málaga: Arguval: 193-196.

Rodríguez de Berlanga, M., 1861, Estudios romanos por él publicados en "La Razón», Madrid: Impr. de Manuel Galiano.

Rodríguez de Berlanga, M., 1864, Monumentos históricos del Municipio Flavio Malacitano, Málaga: El Avisador Malagueño.

Rodríguez de Berlanga, M., 1873 (1995), Los bronces de Osuna [Colección Archivum. Ed. facsímil], Granada: Universidad de Granada.

Rodríguez de Berlanga, M., 1903 (1995), Catálogo del Museo de los Excelentísimos Señores Marqueses de Casa-Loring [ed. facsímil de la de 1903 con estudio preliminar de Pedro Rodríguez Oliva], Málaga: Universidad de Málaga.

Rodríguez Oliva, P., 1991, «Manuel Rodríguez de Berlanga (1825-1909): notas sobre la vida y obra de un estudioso andaluz del mundo clásico», en: J. Arce \& R. Olmos (eds.), Historiografía de la arqueología $y$ de la Historia antigua en España. Madrid: Ministerio de Cultura: 99-106.

Rodríguez Oliva, P., 2001, "Noticias historiográficas sobre el descubrimiento y los primeros estudios en torno a las tablas de bronce con las leyes municipales de Malaca y Salpensa (1861-1864)». Mainake [Monográfico: Las Leyes Municipales en Hispania. 150 Aniversario del descubrimiento de la Lex Flavia Malacitana] XXIII, 9-38.

Rodríguez Oliva, P., 2005, "Un capítulo en las relaciones hispanas de Theodor Mommsen», en: J. Martínez-Pinna (ed.), En el Centenario de Theodoro Mommsen (1817-1903). Homenaje desde la Universidad española, Madrid, Málaga: Real academia de la Historia, Universidad de Málaga: 81-134.

Ruiz Ballón, A., 2013, Pedro Gómez de la Serna (1806-1871). Apuntes para una biografía jurídica, Madrid: Universidad Carlos III de Madrid.

Sociedad Malagueña de Ciencias Físicas y Naturales, 1874, Actas de la Sociedad Malagueña de Ciencias Físicas y Naturales, fundada en sesión de 24 de julio de 1872. Málaga: Imprenta Económica.

Thuiller, L., 1880, "Mapa», www: http://www.idemap.es/malaga/Mapas/CH/SXIX/Plano\%20Málaga\%201880.pdf.

VILA, B., 1861 (1998), Guía del viajero [Edición facsímil], Málaga: Biblioteca Virtual de Andalucía. 